\title{
Investigation of Fatty Acid Ketohydrazone Modified Liposome's Properties as a Drug Carrier
}

\author{
Keita Hayashi, ${ }^{1}$ Madoka Kiriishi, ${ }^{2}$ Keishi Suga, ${ }^{2}$ \\ Yukihiro Okamoto, ${ }^{2}$ and Hiroshi Umakoshi ${ }^{2}$ \\ ${ }^{1}$ Department of Chemical Engineering, National Institute of Technology, Nara College, 22 Yata-cho, Yamatokoriyama, \\ Nara 639-1080, Japan \\ ${ }^{2}$ Division of Chemical Engineering, Graduate School of Engineering Science, Osaka University, 1-3 Machikaneyama-cho, \\ Toyonaka, Osaka 560-8531, Japan \\ Correspondence should be addressed to Hiroshi Umakoshi; umakoshi@cheng.es.osaka-u.ac.jp
}

Received 25 July 2015; Revised 2 October 2015; Accepted 18 October 2015

Academic Editor: Subbu S. Venkatraman

Copyright (C) 2015 Keita Hayashi et al. This is an open access article distributed under the Creative Commons Attribution License, which permits unrestricted use, distribution, and reproduction in any medium, provided the original work is properly cited.

$\mathrm{pH}$-responsive liposomes were prepared by modifying the liposome with acid-cleaving amphiphiles. Palmitic ketohydrazone (P$\mathrm{KH})$ or stearic ketohydrazone (S-KH), composed of hydrophilic sugar headgroup and hydrophobic acyl chain, was used as a modifier of the DMPC liposome. Because the ketohydrazone group of $\mathrm{P}-\mathrm{KH}$ or S-KH was cleaved at low $\mathrm{pH}$ conditions $(<\mathrm{pH}$ 5.0), the delivery of the P-KH modified liposomes was observed probably via an endocytic pathway. The membrane properties of these liposomes were characterized, focusing on the variation of both polarity (measured by Laurdan) and membrane fluidity (measured by DPH) at low $\mathrm{pH}$ condition. The interface of the P-KH modified liposome at acidic $\mathrm{pH}$ was found to become more hydrophobic and less fluidic as compared with that at neutral $\mathrm{pH}$; that is, $\mathrm{P}-\mathrm{KH}$ modified liposome became more rigid structure. Therefore, it seems that the P-KH modified liposome could protect encapsulated drugs from the enzymes in the lysosome. This study shows the novel approach about design of $\mathrm{pH}$-responsive liposomes based on the membrane properties.

\section{Introduction}

Various kinds of self-assembled aggregates, such as vesicle, micelle, and emulsion, are utilized as drug carrier in DDS (drug delivery system) research [1]. The self-assembled aggregates have previously been applied for the capsulation of the drug molecules since the 1980s [2, 3]. In a recent study, liposomes are regarded as one of the frequently used self-assembled aggregates in DDS application [3]. Drugencapsulating liposomes have been already introduced in the medical treatment, for example, Myocet, a liposome encapsulating doxorubicin hydrochloride [4]. Although doxorubicin hydrochloride is treated as an antitumor drug, it is still known to induce strong side effects [5]. Therefore, more efficient encapsulation and targeting strategy are required to reduce side effects of doxorubicin hydrochloride by utilizing liposomes [4].

One of the most contributory factors to reduce side effects is the control of pharmacokinetics, especially, in the case of cancer chemotherapy [6]. Cancer cells are known to secrete some angiogenic growth factors, resulting in the recruitment of disorganized blood vessels to tumor. Disorganized blood vessels allow drug carrier (liposome, etc.) with $\cong 100 \mathrm{~nm}$ diameter to invade to the tumor and to be delivered into the target cell [6]. On the other hand, blood vessels with mature walls prevent the delivery of drug carrier to other normal tissues [6]. The above inhibitory phenomenon has been reported as an enhanced permeability and retention (EPR) effect and, in general, the "passive" strategy to deliver the drugs into tumor cells should be selected by using the "stealth" type drug carrier (e.g., polyethylene glycol modified liposome) [4, 6].

As a post-step of the liposome delivery (approach of liposome into the plasma membrane), the delivery of the drug from liposome into the "cytosol" of the cancer cells is furthermore needed to improve the delivery efficiency [7]. Usually, anticancer drugs including doxorubicin hydrochloride show anticancer activity by binding to DNA of cancer cells [8]. 
The drug cannot be reaching the nuclei unless it could escape from the endosome inside the cytosol [7]. Various kinds of "smart" liposomes have previously been investigated for the escape of drug from endosomal pathway $[9,10]$. The most usual one is $\mathrm{pH}$ stimulus-responsive liposome [9]. It is well known that the $\mathrm{pH}$ condition can be varied from neutral $\mathrm{pH}(\sim 7)$ to acidic $\mathrm{pH}(\sim 5)$ during the endosomal pathway through the contribution of the proton pump of ATPase bound on the membrane [11]. Among the possible smart materials that can make a response against the $\mathrm{pH}$ shift, a hydrazone bond is known as a pH-responsible functional group and its derivatives can be also utilized as the modifier of "smart" liposomes [12].

The key factor to design these liposomes is conventionally known as geometric structure of lipids. For example, phosphatidylethanolamine is frequently used as $\mathrm{pH}$-responsive liposomes [13]. Phosphatidylethanolamine can release proton in low $\mathrm{pH}$ condition [14] and its headgroup can be reduced by the $\mathrm{pH}$ shift [15]. The above variation of the geometric structure at the headgroup of lipid can induce the membrane fusion with the endosomal membrane, resulting in the escape of encapsulated drug from endosome [16]. Although many researchers focus on this $\mathrm{pH}$ shift during the endocytotic pathway, there is no clear strategy for the carrier design, except for the above strategy based on the geometrical structure of carrier molecule.

In this study, the method to design the "membrane surface" of the $\mathrm{pH}$-responsive liposome as "self-assembly" was investigated based on its physicochemical properties. The liposome membrane was modified by a $\mathrm{pH}$-responsive amphiphilic molecule (palmitic ketohydrazone (P-KH) and stearic ketohydrazone $(\mathrm{S}-\mathrm{KH})$ ) that has a ketohydrazone group in between glucose group (headgroup) and acyl chain region [17]. After the membrane properties of the liposome modified with $\mathrm{P}-\mathrm{KH}$ or S-KH were characterized by using the previously reported method [18], the variation of the surface characteristics of the liposome was investigated under the $\mathrm{pH}$ shift. Based on the results on (i) entrapment of drug molecules in the liposome, (ii) its drug delivery efficiency, and (iii) its cytotoxicity, together with the physicochemical properties of the liposome at lower $\mathrm{pH}$, a possibility to apply the $\mathrm{P}-\mathrm{KH}$ liposome and $\mathrm{S}-\mathrm{KH}$ liposome as drug carriers was finally investigated.

\section{Materials and Methods}

2.1. Materials. Phospholipid, 1,2-dimyristoyl-sn-glycero-3phosphatidylcholine (DMPC), was purchased from NOF Corporation (Tokyo, Japan). Fluorescent probes, 1,6-diphenyl-1,3,5-hexatriene (DPH) and 6-dodecanoyl-N,N-dimethyl-2-naphthylamine (Laurdan), were purchased from Sigma Aldrich (St. Louis, MO, USA). D-Glucose was purchased from Kanto Chemical Co., Inc. (Tokyo, Japan). 2,4-Pentanedione was purchased from Wacker Chemie AG (München, Germany). Other chemicals were purchased from Wako Pure Chemical Industries, Ltd. (Osaka, Japan). Chemicals were used without further purification.
2.2. Synthesis of Palmitic Ketohydrazone (P-KH) and Stearic Ketohydrazone $(\mathrm{S}-\mathrm{KH})$. Palmitic ketohydrazone $(\mathrm{P}-\mathrm{KH})$ and stearic ketohydrazone (S-KH) were synthesized by Cglycoside ketone and fatty acid hydrazide as shown in Figure 1 [17]. In order to synthesize C-glycoside ketone, $10 \mathrm{mmol}$ of D-glucose, $10 \mathrm{mmol}$ of sodium carbonate, and $12 \mathrm{mmol}$ of 2,4pentanedione were dissolved in $5 \mathrm{~mL}$ water. This solution was stirred for 4 hours at $90^{\circ} \mathrm{C}$. Reacted solution was washed twice by ethyl acetate to remove the remaining 2,4-pentanedione from the reacted solution. After the aqueous phase was gently collected, C-glycoside ketone was lyophilized. $1.4 \mathrm{mmol}$ of Cglycoside ketone and $2.1 \mathrm{mmol}$ of fatty acid hydrazide were dissolved in $20 \mathrm{~mL}$ of methanol/ethanol $(\mathrm{v} / \mathrm{v}=1: 1)$ and were stirred for 4 hours at $50^{\circ} \mathrm{C}$. After removing the solvent by rotary evaporator, the excessive fatty acid hydrazide was removed by liquid-liquid extraction using water and 4methyl-2-pentanone. Aqueous phase was gently collected, and the water was removed by freeze-drying completely. Obtained $\mathrm{P}-\mathrm{KH}$ and $\mathrm{S}-\mathrm{KH}$ were evaluated by mass spectroscopy, high pressure liquid chromatography, and FTIR to confirm the formation of hydrazone bond.

\subsection{Preparation of P-KH Liposome and S-KH Liposome.} $\mathrm{P}-\mathrm{KH}$ liposome and $\mathrm{S}-\mathrm{KH}$ liposome were prepared by thin film hydration method described previously [18]. 10 $50 \mathrm{~mol} \%$ of P-KH or S-KH was mixed with DMPC in chloroform/methanol. This organic solvent was removed by rotary evaporator. The residual lipid film, after drying under a vacuum overnight, was hydrated with PBS buffer ( $\mathrm{pH}$ 7.3). The liposome suspension was subjected to five cycles of freezing and thawing and was then treated by extrusion device (Liposofast; Avestin Inc., Ottawa, ON, Canada) equipped with two layers of polycarbonate membranes with mean pore diameters of $100 \mathrm{~nm}$.

2.4. Evaluation of Encapsulation Efficiency. Encapsulation efficiency of $\mathrm{P}-\mathrm{KH}$ liposome was evaluated by using a model drug (calcein). P-KH liposome was prepared with $10 \mathrm{mM}$ calcein solution. Nonencapsulated calcein was removed from $\mathrm{P}-\mathrm{KH}$ liposome suspension by gel filtration (Sepharose 4B; GE Healthcare, Little Chalfont, UK). Concentration of DMPC was measured by choline oxidase/DAOS method (Phospholipids C Test Wako). Fluorescence intensity of calcein in the $\mathrm{P}-\mathrm{KH}$ liposome was measured by fluorescence spectrophotometer FP-6500 (Jasco, Tokyo, Japan), after disruption of P-KH liposome by Triton X-100 in order to evaluate strictly encapsulation efficiency without effect of calcein self-quenching [19]. The number of entrapped calcein molecules in a liposome was calculated as follows:

$$
\frac{\text { number of calcein molecules }}{\text { liposome }}=\frac{I_{(+) \text {Triton }}-I_{(-) \text {Triton }}}{C_{\text {lipid }}},
$$

where $I_{(+) \text {Triton }}$ and $I_{(-) T r i t o n}$ represent the fluorescence intensity of each sample after and before Triton X-100 treatment, respectively, and $C_{\text {lipid }}$ is the total concentration of lipid.

2.5. Cell and Cell Culture. Murine rectal cancer cell line (Colon 26 cell) was obtained from RIKEN (RIKEN BRC Cell 


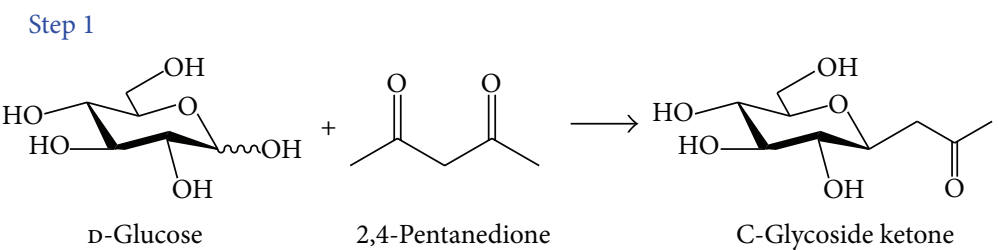

Step 2

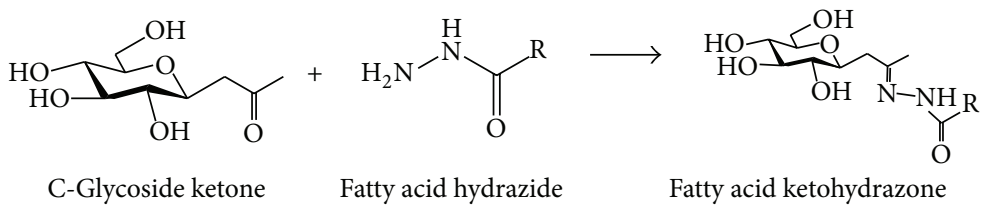

FIGURE 1: Scheme of fatty acid ketohydrazone synthesis.

Bank, Ibaraki, Japan). Colon 26 cells were grown in Eagle's minimal essential medium (E-MEM) (Wako Pure Chemical Industries, Osaka, Japan) supplemented with $10 \%$ fetal bovine serum (FBS) (Thermo Fisher Scientific, Waltham, MA, USA). Colon 26 cells were calculated in the incubator (a humidified atmosphere consisting of $5 \% \mathrm{CO}_{2}$ at $37^{\circ} \mathrm{C}$ ).

2.6. Cytotoxicity Evaluation by MTT Assay. MTT assay was performed by using CellTiter 96 Nonradioactive Cell Proliferation Assay (Promega, Fitchburg, WI, USA) [20]. Colon 26 cells were seeded on the 96-well cell culture plate $\left(100 \mu \mathrm{L}, 2.0 \times 10^{5}\right.$ cells $\left./ \mathrm{mL}\right)$. These cells were cultured for 24 hours in E-MEM supplemented with $10 \%$ FBS in the incubator. A culture medium was exchanged by the new medium $(100 \mu \mathrm{L})$ containing liposomes $(0-5 \mathrm{mM})$. The cells were incubated for 24 hours. $15 \mu \mathrm{L}$ of dye solution was added into the new medium. After incubation for 4 hours in the incubator, $100 \mu \mathrm{L}$ of solubilization solution/stop mix was added into the new medium. After incubation for 1 hour at $37^{\circ} \mathrm{C}, 5 \% \mathrm{CO}_{2}$, an absorbance of each well was measured by microplate spectrophotometer, xMarkTM (BioRad, Hercules, CA, USA).

2.7. Evaluation of Uptake Efficiency by Flow Cytometer and Confocal Laser Microscopy. Cellular uptake of P-KH liposome and $\mathrm{S}-\mathrm{KH}$ liposome was evaluated by flow cytometry. Fluorescence labeled P-KH liposome and S-KH liposome were prepared by mixing with $0.5 \mathrm{~mol} \%$ Rhodamine-PE (Avanti Polar Lipids, Alabaster, AL, USA). Cells were seeded on the 6 -well cell culture plate $\left(1.0 \mathrm{~mL}, 2.0 \times 10^{5}\right.$ cells $\left./ \mathrm{mL}\right)$. Cells were cultured for 24 hours in E-MEM supplemented with $10 \%$ FBS in the incubator. A culture medium was exchanged by the new medium $(1.0 \mathrm{~mL})$ containing fluorescence labeled P-KH liposome and S-KH liposome (5.0 mM). After incubation for 12 hours, the cells were trypsinized and washed twice by PBS. Cells were analyzed by flow cytometer, Attune Acoustic Focusing Cytometer (Applied Biosystems, Inc. Foster City, CA, USA). After incubation for 4 hours, cells were observed by confocal laser microscopy, ECLIPSE TE 2000-U (Nikon, Tokyo, Japan).
2.8. Membrane Fluidity Measurement of DPH. The membrane fluidity of $\mathrm{P}-\mathrm{KH}$ liposome and $\mathrm{S}-\mathrm{KH}$ liposome was evaluated by measuring the fluorescence anisotropy of $\mathrm{DPH}$ (Ex: $360 \mathrm{~nm}, \mathrm{Em}: 430 \mathrm{~nm}$ ) in the liposomal membrane, using a fluorescence spectrophotometer FP-6500 (Jasco, Tokyo, Japan) with a polarizing plate. $10 \mu \mathrm{L}$ of $100 \mu \mathrm{M} \mathrm{DPH}$ in ethanol was added into $1.0 \mathrm{~mL}$ of $0.25 \mathrm{mM}$ P-KH liposome suspension, and the mixture was incubated for $30 \mathrm{~min}$. Polarity $(P)$ was calculated from the following equation:

$$
\begin{aligned}
P & =\frac{I_{0^{\circ} 0^{\circ}}-G I_{0^{\circ} 90^{\circ}}}{I_{0^{\circ} 0^{\circ}}+G I_{0^{\circ} 90^{\circ}}} \\
G & =\frac{I_{90^{\circ} 0^{\circ}}}{I_{90^{\circ} 90^{\circ}}},
\end{aligned}
$$

where $I_{90^{\circ} 0^{\circ}}$ and $I_{90^{\circ} 90^{\circ}}$ are emission intensities perpendicular and parallel to the horizontally polarized light, respectively, and $G$ is the correction factor. Since polarization is inversely proportional to fluidity, membrane fluidity is expressed as $1 / P$.

2.9. Characterization of Hydrophilic/Hydrophobic Membrane Properties by Laurdan. Hydrophilic/hydrophobic characterization of the vesicular membrane was evaluated by an environmentally sensitive fluorescence probe, Laurdan [21]. $1.0 \mu \mathrm{L}$ of $100 \mu \mathrm{M}$ Laurdan (in ethanol) was mixed with $1 \mathrm{~mL}$ of $100 \mu \mathrm{M}$ P-KH liposome or S-KH liposome suspension, and the mixture was incubated for more than $30 \mathrm{~min}$. Laurdan emission spectra from $425 \mathrm{~nm}$ to $550 \mathrm{~nm}$ in the vesicles were obtained by using a fluorescence spectrophotometer FP-6500 (Jasco, Tokyo, Japan), when Laurdan was excited at $340 \mathrm{~nm}$. Thus, the emission spectra were evaluated by calculation of $G P_{(340)}$ value for each emission wavelength as follows:

$$
G P_{(340)}=\frac{\left(I_{440}-I_{490}\right)}{\left(I_{440}+I_{490}\right)},
$$

where $I_{440}$ and $I_{490}$ were fluorescence intensity of Laurdan at $440 \mathrm{~nm}$ and $490 \mathrm{~nm}$, respectively. The values were obtained from the emission spectra using fixed exciting wavelengths of $340 \mathrm{~nm}$. The temperature was kept at $37^{\circ} \mathrm{C}$. 


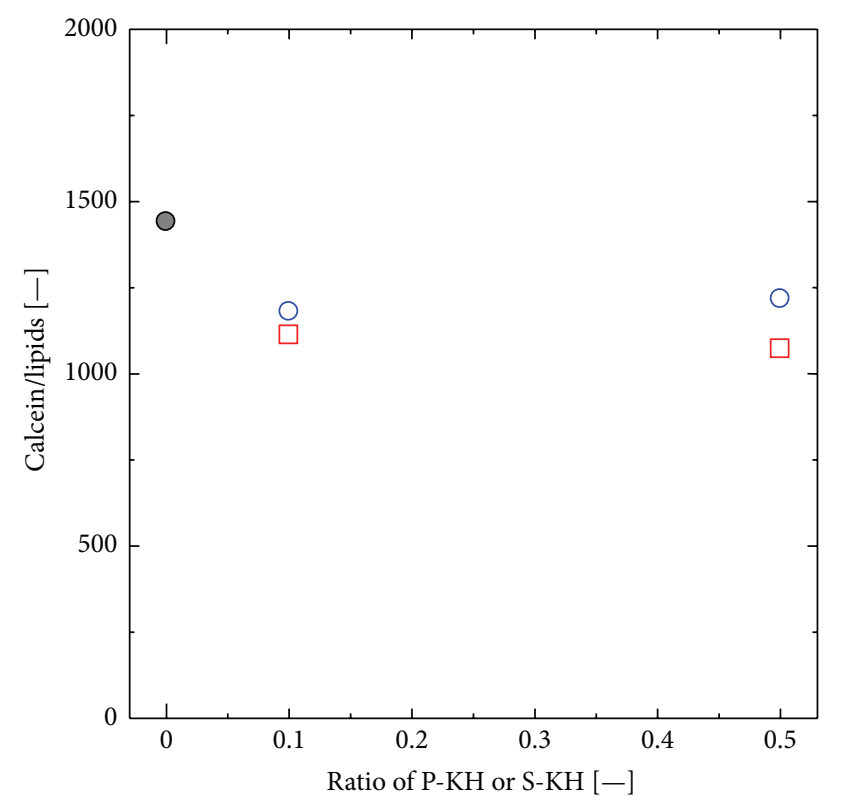

$\square$ DMPC liposome
P-KH liposome
S-KH liposome

FIgURE 2: Entrapment of calcein in the liposomes. P-KH liposome and $\mathrm{S}-\mathrm{KH}$ liposome could encapsulate equal amount of calcein as compared with DMPC liposome.

\section{Results}

3.1. Drug Encapsulation, Drug Delivery Efficiency, and Cytotoxicity of $\mathrm{P}-\mathrm{KH}$ Liposome and $\mathrm{S}-\mathrm{KH}$ Liposome. Entrapment of model drug (calcein) in $\mathrm{P}-\mathrm{KH}$ liposome and S-KH liposome was evaluated by monitoring its fluorescence intensity. The number of calcein molecules per liposome was calculated as shown in Figure 2. The number of calcein molecules in the DMPC liposome was $1441 \mathrm{~mol}$-calcein/mol-lipid, and those in the 10\% P-KH liposome and 10\% S-KH liposome were 1112 units and 1181 units, respectively. Although the number of calcein molecules in the P-KH liposome and S-KH liposome was slightly lower than that in DMPC liposome, P-KH liposome and $\mathrm{S}-\mathrm{KH}$ liposome were found to encapsulate the calcein molecules inside their inner water pool. Moreover, the increase of the molar ratio of $\mathrm{P}-\mathrm{KH}$ and S-KH hardly affected the numbers of calcein molecules in the liposomes, implying that the DMPC liposomes were successfully modified with P$\mathrm{KH}$ and $\mathrm{S}-\mathrm{KH}$ within the modifier ratio of $0-50 \%$.

In order to evaluate the drug delivery efficiency, the uptake of three kinds of liposomes (such as DMPC liposome, $\mathrm{P}-\mathrm{KH}$ liposome, and S-KH liposome) by colon 26 cells was evaluated by using flow cytometer (Figure 3(a)). This result shows that colon 26 cells took up similar amount of these liposomes. Therefore, both $\mathrm{P}-\mathrm{KH}$ liposome and $\mathrm{S}-\mathrm{KH}$ liposome can deliver the drug to the inside of cells and can also be applied as a drug carrier. Moreover, colon 26 cells were observed by confocal laser microscopy when colon 26 cells were treated with fluorescence labeled P-KH liposome (Figure 3(b)). Red fluorescence was observed at the inside of the cells. This result shows that colon 26 cells took up the P$\mathrm{KH}$ liposome by endocytotic mechanism, judging from our previous report [20].

The cytotoxicity of P-KH liposome and S-KH liposome is an important factor to apply them as a drug carrier. The cytotoxicity of the liposomes (DMPC liposome, P$\mathrm{KH}$ liposome, and S-KH liposome) for colon 26 cells was evaluated by MTT assay. Figure 4 shows the viability of colon 26 cells after their coincubation with DMPC liposome, P$\mathrm{KH}$ liposome, or S-KH liposome. DMPC liposome and S-KH liposome hardly showed cytotoxic effect for colon 26 cells, although the cell viability of colon 26 cells was reduced to $\sim 80 \%$ by the treatment with S-KH liposome. On the other hand, the viability of colon 26 cells was significantly reduced by P-KH liposome (10\% and 30\%) and reached less than $40 \%$. It was thus investigated that only $\mathrm{P}-\mathrm{KH}$ liposome showed cytotoxicity for colon 26 cells.

3.2. Variation of Membrane Properties of P-KH Liposome and $S$-KH Liposome at Lower $p H$ Condition. It is well known that the proton pump of a membrane bound ATPase can transport proton across the endosomal membrane, suggesting that the $\mathrm{P}-\mathrm{KH}$ liposome or S-KH liposome could be exposed to acidic $\mathrm{pH}$ in their use in the endosomal environment. In order to evaluate the $\mathrm{pH}$ effect on the membrane surface of $\mathrm{P}-\mathrm{KH}$ liposome and $\mathrm{S}-\mathrm{KH}$ liposome, the membrane properties of the liposomes were measured in lower $\mathrm{pH}$ conditions.

Figure 5 shows $\mathrm{pH}$ dependence of the membrane fluidity $(1 / P$ value $)$ and hydrophilic/hydrophobic environment (membrane polarity, $G P_{(340)}$ value) of the liposomes. The $1 / P$ values of $\mathrm{P}-\mathrm{KH}$ liposomes decreased at $\mathrm{pH} 5$, while the $G P_{(340)}$ values of $\mathrm{P}-\mathrm{KH}$ liposomes increased at $\mathrm{pH} 5$. It has been reported that a hydrazone bond is stable at $\mathrm{pH} \sim 7$, and it turns out to be cleaved at lower $\mathrm{pH}$ less than 5 [22]. Therefore, $\mathrm{P}-\mathrm{KH}$ was assumed to be cleaved to $\mathrm{C}$-glycoside ketone and palmitic acid hydrazide at $\mathrm{pH} \mathrm{5,} \mathrm{where} \mathrm{the} \mathrm{P-KH} \mathrm{molecules} \mathrm{on} \mathrm{the}$ liposomes can be partly altered to palmitic acid. The variation of the P-KH composition on the liposome membrane could induce the alteration of the membrane properties during the $\mathrm{pH}$ shift. This type of $\mathrm{pH}$ shift is, in general, observed inside the cell during its endocytotic pathway. Although the membrane properties of S-KH liposome did not show significant alteration by $\mathrm{pH}$ gradient, it is notable that the membrane fluidities of liposomes increased by modification both with P-KH and with S-KH.

\section{Discussion}

In this study, the P-KH liposome and S-KH liposome were prepared as $\mathrm{pH}$-responsive drug carrier and their membrane properties were analyzed by using fluorescent probes. In recent years, various kinds of stimulus-responsive drug carrier were investigated in order to deliver drug effectively for diseased cells $[9,10]$. A pH gradient is one of the most available stimulations for controlled release of drugs [9]. Some kinds of $\mathrm{pH}$-responsive drug carriers have been previously investigated [7]. Here, P-KH liposomes and S-KH liposomes were characterized as the $\mathrm{pH}$-responsive drug carriers, and the membrane properties, such as membrane fluidity $(1 / P)$ 


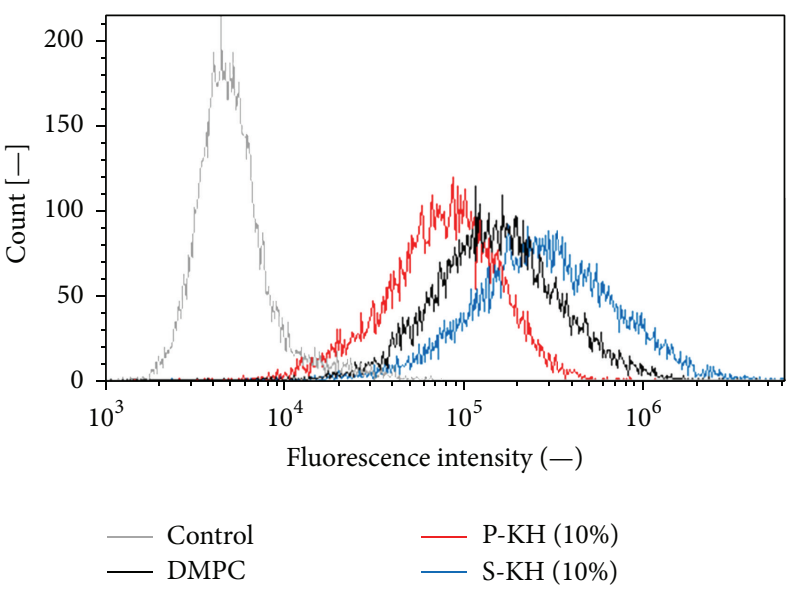

(a)
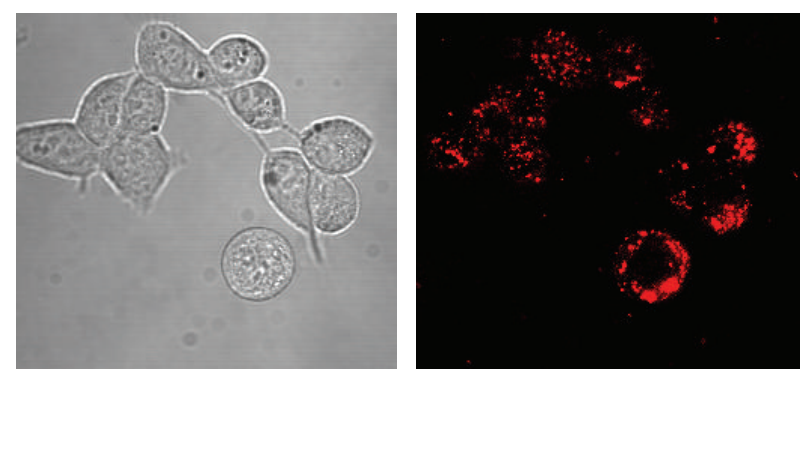

(b)

FIGURE 3: Drug delivery efficiency of P-KH liposome and S-KH liposome. (a) Quantitative evaluation of drug delivery efficiency by flow cytometer. (b) Images of confocal laser microscopy of colon 26 cells which were treated with fluorescence labeled P-KH liposome.

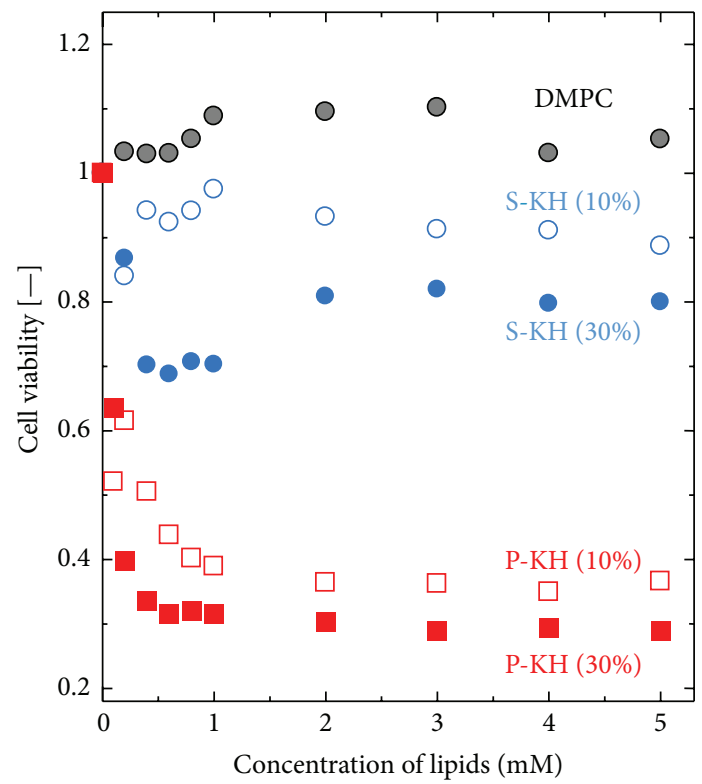

Figure 4: Cytotoxic effect of P-KH liposome and S-KH liposome on colon 26 cells. High concentration of P-KH liposome shows cytotoxicity effect on colon 26 cells. It is important to control a concentration of $\mathrm{P}-\mathrm{KH}$ liposome in order to be used as a drug carrier.

and membrane polarity $\left(G P_{(340)}\right)$, were investigated at lower $\mathrm{pH}$ condition that can imitate the endosomal condition. The membrane properties of the liposome could be related to its feature as a drug carrier; for example, the membrane fluidity of liposome significantly relates to the drug release from the liposomal inner phase [19]. When the $\mathrm{pH}$ value of $\mathrm{P}-\mathrm{KH}$ liposome suspension can alter from $\mathrm{pH} 7$ to $\mathrm{pH} 5$, the $1 / P$ values of $\mathrm{P}-\mathrm{KH}$ liposomes decreased and the $G P_{(340)}$ values increased, indicating that the $\mathrm{P}-\mathrm{KH}$ liposome membranes turned out to be rigid at $\mathrm{pH}$ 5. These characteristics of $\mathrm{P}-\mathrm{KH}$ liposomes will contribute to predicting the behaviors of $\mathrm{P}-\mathrm{KH}$ liposome in the endosome.
However, S-KH liposomes indicated no clear variation of membrane properties by $\mathrm{pH}$ gradient, though the chemical structure of S-KH is very similar to that of P-KH. In general, the phase transition temperature of DSPC liposome, composed of "stearic acid," from $\mathrm{L}_{\beta}$ phase to $\mathrm{P}_{\beta}$ phase is known to be $49.1^{\circ} \mathrm{C}$ and that from $\mathrm{P}_{\beta}$ phase to $\mathrm{L}_{\alpha}$ phase is $54.5^{\circ} \mathrm{C}$ [23]. In addition, the value of DPPC liposome, composed of "palmitic acid," from $\mathrm{L}_{\beta}$ phase to $\mathrm{P}_{\beta}$ phase is $34.4^{\circ} \mathrm{C}$ and that from $\mathrm{P}_{\beta}$ phase to $\mathrm{L}_{\alpha}$ phase is $41.3^{\circ} \mathrm{C}$ [23]. The phase transition temperature of "stearic acid-based (S-based)" lipid seems to be higher than that of "palmitic acid-based (P-based)" lipid, implying the "relatively rigid" nature of "S-based" lipid. It is therefore suggested that the S-KH and P-KH could also show different behaviors at the interior of the membrane of "self-assembly (liposome)." The phase transition temperature of DMPC liposome is known to be $23.6^{\circ} \mathrm{C}$ [23], so that the phase separation of "relatively rigid" S-KH could occur on the "relatively fluid" DMPC liposome membrane at $37^{\circ} \mathrm{C}$. The region of "relatively rigid" S-KH phase on the DMPC liposome membrane is not easily affected by $\mathrm{pH}$ gradation as compared with $\mathrm{P}-\mathrm{KH}$ owing to the less flexibility of lipid molecules around the "relatively rigid" region. Therefore, P$\mathrm{KH}$ liposomes turned out to be rigid, while S-KH liposomes showed higher membrane fluidities as compared to DMPC liposome.

It seems that the variation of the membrane properties of $\mathrm{P}-\mathrm{KH}$ liposomes could be caused by the cleavage of hydrazone bond that conjugating with headgroup (glucose-glycoside ketone) and hydrophobic tail (palmitic acid hydrazide). The reduction of $\mathrm{pH}$ altered the partial amount of $\mathrm{P}-\mathrm{KH}$ molecules to palmitic acid hydrazide on the liposome surface. It has been reported that the composition of vesicle including liposome directly affects the membrane properties of vesicles [24]. Han has previously reported that the diffusion coefficient of fatty acid was different from that of phospholipid on the lipid bilayer membrane by molecular dynamic simulation, in spite of same acyl chain structure between fatty acid and phospholipid [25]. Focusing on the membrane-membrane 

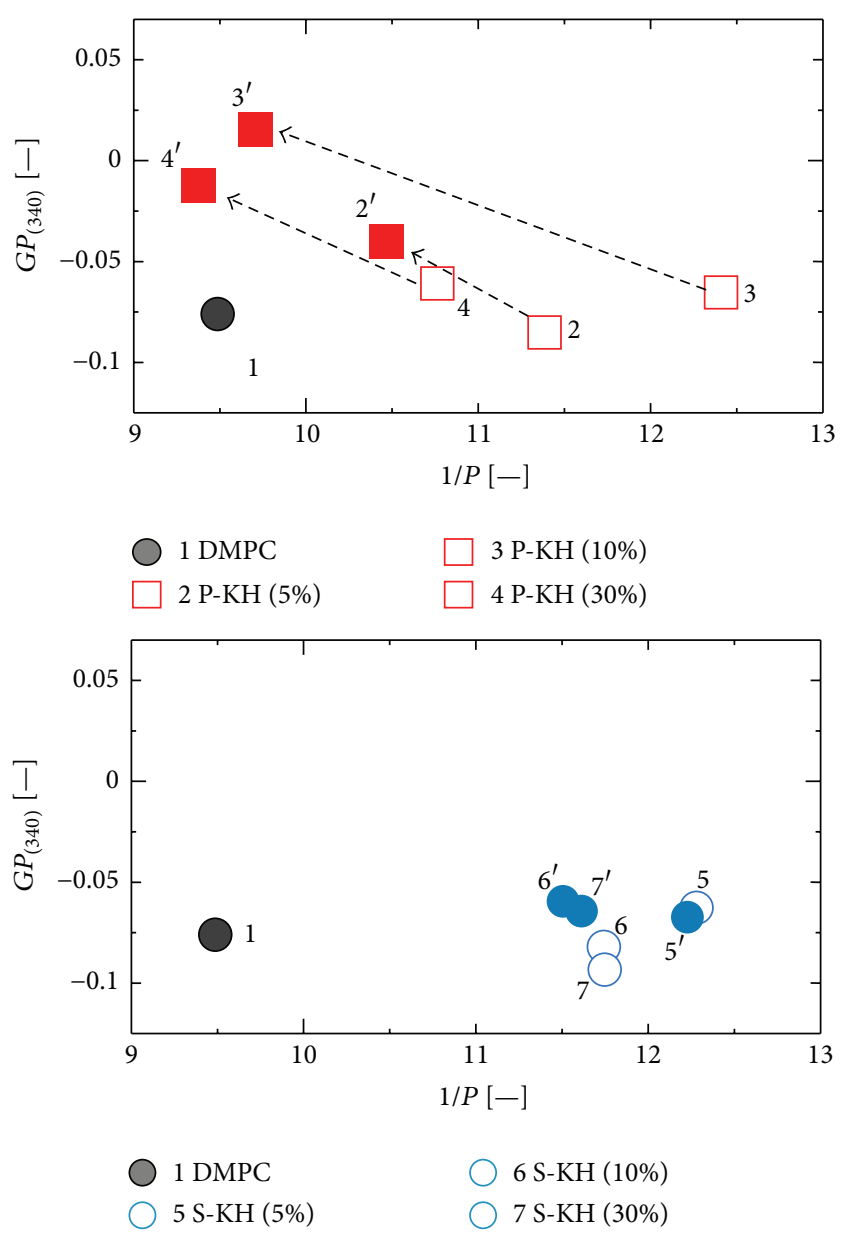

FIgURE 5: Alteration of membrane properties with dropping $\mathrm{pH}$. Membrane properties were evaluated by membrane fluidity $(1 / P$, anisotropy of DPH fluorescence) and hydrophilic/hydrophobic environment $\left(G P_{(340)}\right.$ values calculated by Laurdan fluorescence intensity) of liposomal membrane. 2 4 and 5 7 show the physicochemical properties of $\mathrm{P}-\mathrm{KH}$ liposome and $\mathrm{S}-\mathrm{KH}$ liposome in PBS, and $2^{\prime} \sim 4^{\prime}$ and $5^{\prime} \sim 7^{\prime}$ show that of P-KH liposome and S-KH liposome at $\mathrm{pH} 5.0$.

interaction, a higher headgroup mobility of Span 80 vesicle could be a possible key to induce the perturbation of target membrane [24]. It seems that the headgroup structure was important factor to control the membrane properties and functions. Therefore, P-KH liposome can be used as a smart ( $\mathrm{pH}$ variable) drug carrier based on membrane properties by $\mathrm{pH}$ stimulation.

In the case of possible application of $\mathrm{P}-\mathrm{KH}$ liposome in actual DDS, the cytotoxicity of P-KH liposome could also be a potential problem. In this study, P-KH liposome at the higher concentration shows the cytotoxicity for colon 26 cells (Figure 4). The results imply that there could be a possible problem of applicability of its $\mathrm{P}-\mathrm{KH}$ liposome as a gene carrier although further investigation is needed to explain the cytotoxicity of P-KH liposome not only in vitro but also in vivo. The above findings also show that the $\mathrm{P}-\mathrm{KH}$ liposome itself has a potential to function as an antitumor agent. It seems that the "smart" characteristics of the P-KH liposome surface could be utilized for its use against a solid tumor, considering the EPR effect [26].

\section{Conflict of Interests}

The authors declare that there is no conflict of interests regarding the publication of this paper.

\section{Acknowledgment}

This research was supported by a Grant-in-Aid for Research Activity Start-Up (no. 25889065) and a Grant-in-Aid for Scientific Research (A) (no. 26249116) from the Japan Society for the Promotion of Science (JSPS).

\section{References}

[1] Y. Zhai and G. Zhai, "Advances in lipid-based colloid systems as drug carrier for topic delivery," Journal of Controlled Release, vol. 193, pp. 90-99, 2014.

[2] S. Wiedersberg and R. H. Guy, "Transdermal drug delivery: 30 + years of war and still fighting!, Journal of Controlled Release, vol. 190, pp. 150-156, 2014.

[3] J. V. Natarajan, C. Nugraha, X. W. Ng, and S. Venkatraman, "Sustained-release from nanocarriers: a review," Journal of Controlled Release, vol. 193, pp. 122-138, 2014.

[4] K. Mross, B. Niemann, U. Massing et al., "Pharmacokinetics of liposomal doxorubicin (TLC-D99; Myocet) in patients with solid tumors: an open-label, single-dose study," Cancer Chemotherapy and Pharmacology, vol. 54, no. 6, pp. 514-524, 2004.

[5] S. E. Lipshultz, S. D. Colan, R. D. Gelber, A. R. Perez-Atayde, S. E. Sallan, and S. P. Sanders, "Late cardiac effects of doxorubicin therapy for acute lymphoblastic leukemia in childhood," The New England Journal of Medicine, vol. 324, no. 12, pp. 808-815, 1991.

[6] K. Maruyama, "Intracellular targeting delivery of liposomal drugs to solid tumors based on EPR effects," Advanced Drug Delivery Reviews, vol. 63, no. 3, pp. 161-169, 2011.

[7] O. V. Gerasimov, J. A. Boomer, M. M. Qualls, and D. H. Thompson, "Cytosolic drug delivery using $\mathrm{pH}$ - and light-sensitive liposomes," Advanced Drug Delivery Reviews, vol. 38, no. 3, pp. 317-338, 1999.

[8] M. Verrill, "Anthracyclines in breast cancer: therapy and issues of toxicity," The Breast, vol. 10, supplement 2, pp. 8-15, 2001.

[9] C.-S. Lee, W. Park, S.-J. Park, and K. Na, "Endolysosomal environment-responsive photodynamic nanocarrier to enhance cytosolic drug delivery via photosensitizer-mediated membrane disruption," Biomaterials, vol. 34, no. 36, pp. 9227-9236, 2013.

[10] I. Levacheva, O. Samsonova, E. Tazina et al., "Optimized thermosensitive liposomes for selective doxorubicin delivery: formulation development, quality analysis and bioactivity proof," Colloids and Surfaces B: Biointerfaces, vol. 121, pp. 248-256, 2014.

[11] M. Wienisch and J. Klingauf, "Vesicular proteins exocytosed and subsequently retrieved by compensatory endocytosis are nonidentical," Nature Neuroscience, vol. 9, no. 8, pp. 1019-1027, 2006.

[12] A. A. Kale and V. P. Torchilin, “'Smart' drug carriers: PEGylated TATp-modified pH-sensitive liposomes," Journal of Liposome Research, vol. 17, no. 3-4, pp. 197-203, 2007. 
[13] H. Farhood, N. Serbina, and L. Huang, "The role of dioleoyl phosphatidylethanolamine in cationic liposome mediated gene transfer," Biochimica et Biophysica Acta, vol. 1235, no. 2, pp. 289295, 1995.

[14] M. R. Moncelli, L. Becucci, and R. Guidelli, "The intrinsic pKa values for phosphatidylcholine, phosphatidylethanolamine, and phosphatidylserine in monolayers deposited on mercury electrodes," Biophysical Journal, vol. 66, no. 6, pp. 1969-1980, 1994.

[15] A. D. Petelska, M. Naumowicz, and Z. A. Figaszewski, "The influence of $\mathrm{pH}$ on phosphatidylethanolamine monolayer at the air/aqueous solution interface," Cell Biochemistry and Biophysics, vol. 65, no. 2, pp. 229-235, 2013.

[16] Y. Xu and F. C. Szoka Jr., "Mechanism of DNA release from cationic liposome/DNA complexes used in cell transfection," Biochemistry, vol. 35, no. 18, pp. 5616-5623, 1996.

[17] F. Rodrigues, Y. Canac, and A. Lubineau, "A convenient, onestep, synthesis of $\beta$-C-glycosidic ketones in aqueous media," Chemical Communications, no. 20, pp. 2049-2050, 2000.

[18] K. Hayashi, T. Shimanouchi, K. Kato, T. Miyazaki, A. Nakamura, and H. Umakoshi, "Span 80 vesicles have a more fluid, flexible and "wet" surface than phospholipid liposomes," Colloids and Surfaces B: Biointerfaces, vol. 87, no. 1, pp. 28-35, 2011.

[19] T. Shimanouchi, H. Ishii, N. Yoshimoto, H. Umakoshi, and R. Kuboi, "Calcein permeation across phosphatidylcholine bilayer membrane: effects of membrane fluidity, liposome size, and immobilization," Colloids and Surfaces B: Biointerfaces, vol. 73, no. 1, pp. 156-160, 2009.

[20] K. Hayashi, T. Tatsui, T. Shimanouchi, and H. Umakoshi, "Enhanced cytotoxicity for Colon 26 cells using Doxorubicinloaded sorbitan monooleate (Span 80 ) vesicles," International Journal of Biological Sciences, vol. 9, no. 2, pp. 142-148, 2013.

[21] T. Parasassi, M. Di Stefano, M. Loiero, G. Ravagnan, and E. Gratton, "Influence of cholesterol on phospholipid bilayers phase domains as detected by Laurdan fluorescence," Biophysical Journal, vol. 66, no. 1, pp. 120-132, 1994.

[22] R. Patil, J. Portilla-Arias, H. Ding et al., "Cellular delivery of doxorubicin via $\mathrm{pH}$-controlled hydrazone linkage using multifunctional nano vehicle based on poly( $\beta$-L-malic acid)," International Journal of Molecular Sciences, vol. 13, no. 9, pp. 11681-11693, 2012.

[23] R. Koynova and M. Caffrey, "Phases and phase transitions of the phosphatidylcholines," Biochimica et Biophysica Acta, vol. 1376, no. 1, pp. 91-145, 1998.

[24] K. Hayashi, T. Tatsui, T. Shimanouchi, and H. Umakoshi, "Membrane interaction between Span 80 vesicle and phospholipid vesicle (liposome): Span 80 vesicle can perturb and hemifuse with liposomal membrane," Colloids and Surfaces B: Biointerfaces, vol. 106, pp. 258-264, 2013.

[25] S. Han, "Molecular dynamics simulation of oleic acid/oleate bilayers: an atomistic model for a ufasome membrane," Chemistry and Physics of Lipids, vol. 175-176, pp. 79-83, 2013.

[26] H. Maeda, "The enhanced permeability and retention (EPR) effect in tumor vasculature: the key role of tumor-selective macromolecular drug targeting," Advances in Enzyme Regulation, vol. 41, no. 1, pp. 189-207, 2001. 

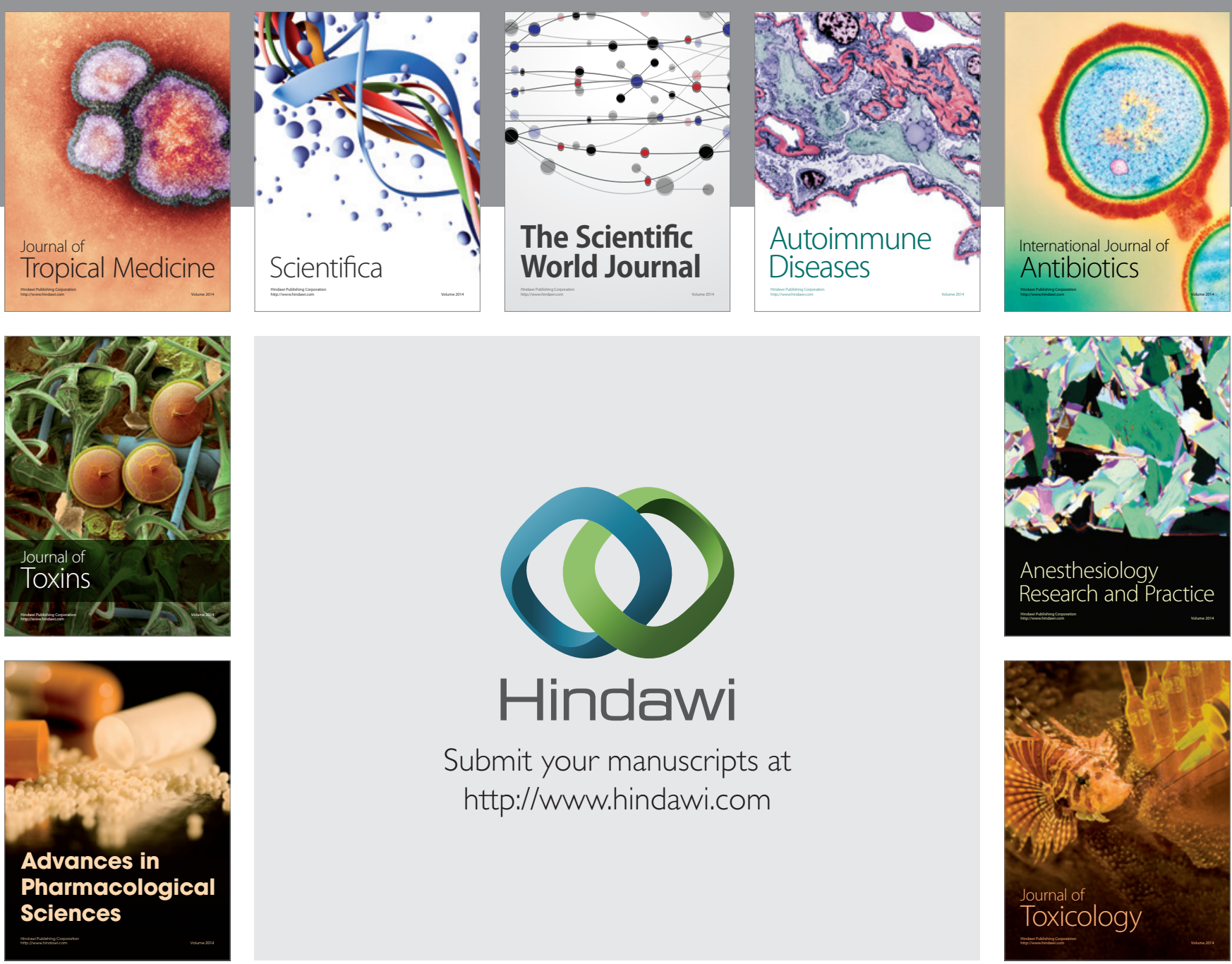

\section{Hindawi}

Submit your manuscripts at

http://www.hindawi.com
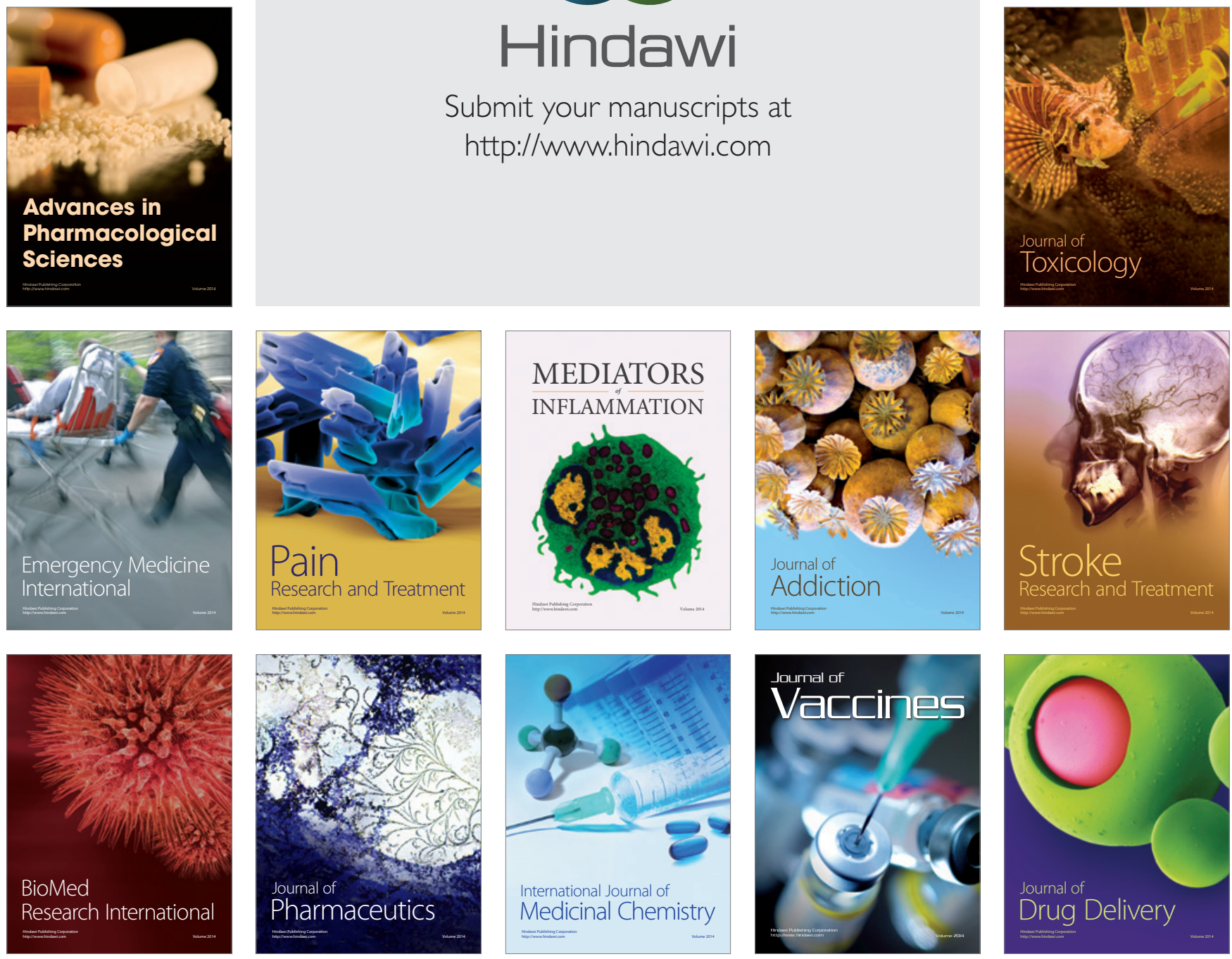